\title{
Safety and Tolerability of the Potassium Binder Patiromer From a Global Pharmacovigilance Database Collected Over 4 Years Compared with Data from the Clinical Trial Program
}

\author{
Patrick Rossignol ${ }^{1,2} \cdot$ Lea David $^{3} \cdot$ Christine Chan $^{3} \cdot$ Ansgar Conrad $^{3} \cdot$ Matthew R. Weir $^{4}$
}

Accepted: 22 April 2021 / Published online: 20 May 2021

(c) The Author(s) 2021

\begin{abstract}
Introduction The availability of the sodium-free potassium binder patiromer opens new opportunities for hyperkalemia management.

Objective Our objective was to compare data from a 4-year global pharmacovigilance database of adverse events (AEs) reported in patients prescribed patiromer in clinical practice compared with data obtained from the clinical trial program.

Methods Postmarketing safety data regarding patiromer (Veltassa ${ }^{\circledR}$; Vifor Pharma, Inc.), collected and recorded in the company's global pharmacovigilance database, were analyzed for the period from January 2016 through September 2019. These data were both solicited (i.e., via an organized data-collection method such as a patient-support program) and unsolicited (i.e., voluntarily reported by healthcare professionals, consumers, and competent authorities worldwide). The cumulative annualized mortality rate (events per 100 patient-years [PYs]) for the pharmacovigilance database analysis period were compared with the rate obtained in the longest patiromer clinical trial to date (up to 52 weeks of treatment). For individual AEs, reporting rates (\% of events/100 PYs) for events collected in the global pharmacovigilance database were compared with the frequencies (\% of patients with event/patients exposed) of events collected in the clinical trial program $(N=666)$. Results Over 4 years, the global pharmacovigilance database contained an estimated 45,000 PYs of exposure $(17,823$ individual case reports and 38,109 AEs), with most cases (95\%) from the USA; > 85\% of cases utilized $8.4 \mathrm{~g} /$ day. In total, 1214 deaths were reported, with a cumulative annualized mortality rate of 2.69/100 PYs (vs. 5.70 deaths/100 PYs in the 52-week clinical trial). Global pharmacovigilance reporting rates for the two most common AEs, constipation and diarrhea, were 6.90 and $3.48 \%$, respectively. Respective frequencies were 7.2 and $4.8 \%$ in the clinical trial program. The pharmacovigilance reporting rate for AEs of decreased blood potassium was $0.45 \%$; serum potassium $<3.5 \mathrm{mmol} / \mathrm{L}$ was reported in $4.7 \%$ of patients in the clinical trial program. For hypomagnesemia or decreased blood magnesium, reporting rates in the postmarketing setting were 0.02 and $0.16 \%$, respectively, and they were observed in 5.3 and $0.8 \%$ of patients, respectively, in the clinical trial program.

Conclusions Global pharmacovigilance data over 4 years confirmed that the tolerability and safety of patiromer in clinical practice is predictable and consistent with clinical trial data, with no evidence of any new safety signals to date.
\end{abstract}

Patrick Rossignol

p.rossignol@chru-nancy.fr

1 University of Lorraine, Inserm 1433 CIC-P CHRU de

Nancy, Inserm U1116, Nancy, France

2 F-CRIN INI-CRCT, Nancy, France

3 Vifor Pharma, Inc., Redwood City, CA, USA

4 University of Maryland School of Medicine, Baltimore, MD, USA 


\section{Key Points}

Patiromer (Veltassa ${ }^{\circledR}$, Vifor Pharma, Inc.) is a sodiumfree, nonabsorbed, potassium binder that was initially approved by the US FDA in 2015 and in the EU/European Economic Area in 2017 and is now approved in many countries worldwide.

Reported adverse events from patients prescribed patiromer for hyperkalemia management collected between January 2016 and September 2019 in the global pharmacovigilance database were analyzed; the total number of individual case reports was 17,823 .

The tolerability and safety profile of patiromer in clinical practice was consistent with that observed in clinical trials, supporting a predictable risk-benefit profile with no evidence of any new safety signals to date.

\section{Introduction}

The availability in the USA [1], the EU [2], and other countries of the sodium-free, nonabsorbed, potassium-binding polymer patiromer (Veltassa ${ }^{\circledR}$, Vifor Pharma, Inc.) provides new opportunities for hyperkalemia management across the cardiovascular and renal continuum [3]. Older-generation potassium binders (i.e., sodium or calcium polystyrene sulfonate [SPS or CPS]) were approved decades ago when the concept of evidence-based medicine was not yet fully adopted. The tolerability of SPS is generally poor, and it has been associated with fluid overload, which may be due to the sodium content [3], and rare but severe colonic necrosis [4, 5]. Newer potassium binders such as patiromer and sodium zirconium cyclosilicate [6] have the potential to be used for the persistent or chronic treatment of hyperkalemia, thereby allowing patients with chronic kidney disease (CKD), diabetes, or heart failure (HF) who require treatment with guideline-directed renin-angiotensin-aldosterone system inhibitors (RAASi) to remain on these life-saving therapies [7-9].

Patiromer has been shown to enable concomitant RAASi treatment in patients being treated for hyperkalemia. In the randomized withdrawal phase of the OPAL-HK study, 52\% of patients who switched to placebo discontinued RAASi treatment because of recurrent hyperkalemia compared with $5 \%$ of patients who continued treatment with patiromer [10]. Placebo-controlled studies [11, 12] have also shown patiromer's efficacy in preventing hyperkalemia and thereby enabling the use of spironolactone. More recently, consensus recommendations on the newer potassium binders (patiromer and sodium zirconium cyclosilicate) from the Heart Failure Association of the European Society of Cardiology noted that these agents may be considered to manage hyperkalemia in select patients with HF, with or without CKD, to enable use of mineralocorticoid receptors and other RAASi at higher doses [13]. The Kidney Disease: Improving Global Outcomes (KDIGO) 2020 clinical practice guideline for diabetes management in CKD also proposed potassium binders as one of a number of measures to manage hyperkalemia associated with the use of RAASi [14]. However, it is not currently known whether this approach will improve patient outcomes.

The two most common treatment-emergent adverse events (AEs; occurring in $\geq 5 \%$ of patients) in patients treated with patiromer in the clinical trial program were constipation (7.2\%) and hypomagnesemia (5.3\%) [1]. Constipation generally resolved during the course of treatment. Most AEs were mild to moderate, and no serious AEs that were considered related to patiromer treatment were reported. Approximately $4.7 \%$ of patients developed serum potassium $<3.5 \mathrm{mmol} / \mathrm{L}$, and roughly $9 \%$ of patients developed serum magnesium $<$ $0.58 \mathrm{mmol} / \mathrm{L}$ [1]. These data were derived from four clinical trials $[10,11,15,16]$ in which patients were initiated on patiromer in divided doses between 8.4 and $33.6 \mathrm{~g}$ daily. Real-world data indicate that the most widely used patiromer starting dose in clinical practice is $8.4 \mathrm{~g}$ daily $[17,18]$.

Because clinical trials are conducted under widely varying conditions, AE rates may not reflect those observed in practice [1]. In this paper, we describe the reporting rate of AEs observed during the post-approval use of patiromer, using the global pharmacovigilance database of the patiromer manufacturer, and compare these with data from the clinical trial program.

\section{Methods}

\subsection{Data Sources}

Postmarketing safety data regarding patiromer (Veltassa ${ }^{\circledR}$ ) were collected and recorded in the company's global pharmacovigilance database. The data recorded in the global pharmacovigilance database were analyzed for the period from January 2016 through September 2019. The majority of AEs were from the USA (95\%), and the remainder came from Austria, Belgium, Denmark, Finland, Germany, Israel, Netherlands, Norway, Spain, Switzerland, Saudi Arabia, UK, and Uruguay. From a pharmacovigilance perspective, an AE can be any unfavorable or unintended sign, symptom, or disease temporally related with the use of a medicinal product, whether or not it is related to the medicinal product [19]. In addition, the US FDA indicated that a report or information submitted by a reporter does 
not necessarily reflect a conclusion that the report or information constitutes an admission that the drug caused or contributed to an AE [20]. Temporal association is defined as events associated in time but not necessarily linked as cause and effect. The Uppsala Monitoring Centre, which is a part of the World Health Organization (WHO) Collaborating Centre for International Drug Monitoring, holds guidance on causality assessments. The company's pharmacovigilance database uses the WHO-Uppsala Monitoring Centre Standardized Causality Assessment System to assess the relationship between AEs and drugs [21]. The global pharmacovigilance database consists of $\mathrm{AE}$ reports obtained in both a solicited and an unsolicited (spontaneous) manner. Solicited reports are defined as those obtained from an organized data-collection method (including, but not limited to, patient-support programs, disease-management programs, surveys of patients by healthcare providers, observational studies, and interactions between healthcare providers and company representatives), and all AEs regardless of whether the relationship with patiromer was mentioned within the exchange were captured. The patient-support program provides a single point of contact to help the patient quickly start patiromer and stay on therapy. This enables the patient and prescriber to obtain easier access to the product, including refills, reimbursement, insurance verification and approval, and all necessary documentation needed to fill prescriptions. Unsolicited (spontaneous) AEs are defined as those voluntarily reported by healthcare professionals, consumers, and competent authorities worldwide (e.g., FDA, European Medicines Agency [EMA]). All information obtained from a healthcare provider (e.g., physician, pharmacist, nurse) are considered medically confirmed; however, these reports may not signify information that confirms a suspected relationship between the drug and the reported AE.

In addition, a systematic literature and monitoring review of widely used reference databases, such as Embase $^{\circledR}$ (Elsevier) and PubMed (US National Library of Medicine), with a defined search string of any mention of "patiromer" or "Veltassa" or "RLY5016," is used to identify public reports of adverse drug reaction (ADR) cases and record these in the global pharmacovigilance database. All ADRs are analyzed to determine whether a possible change to the benefit-risk profile of patiromer is needed and, in particular, to detect potential new safety signals, including AEs that have been identified in any literature search.

All cases reported in the pharmacovigilance database were medically reviewed for clinical content, which includes medical history, concomitant medications, and possible causal relationship of AE to drug using the WHO-Uppsala Monitoring Centre Standardized Causality Assessment
System. Further, three follow-up attempts are made to obtain information.

\subsection{Data Collection}

The collection and the analysis of data retrieval, which includes duplicate checks, is a required standard pharmacovigilance process across the industry. Drug safety physicians review and analyze all individual case safety reports (ICSRs). Safety data were structured by ICSRs that could include one or more AEs in a single patient (i.e., number of events is not equivalent to number of patients; each ICSR is one patient). Although individual patients may have multiple events reported in the database, the timing of some of these events is unknown. In addition, because of confidentiality rules or limited information reported on patients, a given $\mathrm{AE}$ may have been reported multiple times from different sources with a limited possibility for reconciliation and duplicate removal. Verbatim AE terms from solicited and unsolicited sources from medical and nonmedical reporters are coded according to the most appropriate preferred terms defined by the Medical Dictionary for Regulatory Activities (MedDRA V22.1). For AEs associated with laboratory abnormalities (e.g., "blood potassium decreased," hypomagnesemia, etc.), quantitative measures to confirm the accuracy of the reported AE term are inconsistent. Therefore, the reporting of these AEs is less rigorous than in clinical trials, where such quantitative laboratory data are available. In addition, the patiromer clinical trials had an independent adjudication committee that evaluated all deaths that occurred in the studies. In the global postmarketing database, no adjudication was conducted other than follow-up to obtain further information.

\subsection{Data Analysis}

Data were analyzed using summary tables of all AEs and all serious AEs generated from the global pharmacovigilance database to identify any emerging events, unusual trends, or reporting frequencies. An AE is considered serious if it meets one or more of the following criteria:

1. Results in death or is life threatening.

2. Requires inpatient hospitalization or prolongation of existing hospitalization.

3. Results in persistent or significant disability or incapacity.

4. Results in a congenital anomaly (birth defect).

5. Is otherwise "medically significant" (e.g., does not meet the preceding criteria but is considered serious because treatment/intervention would be required to prevent one of the preceding criteria). 
In addition, any AEs identified in the list of important medical events [22] (a list created by the EMA for the review of suspected AEs that deserve special attention) were upgraded as a serious AE in the global pharmacovigilance database. Duplicate reports were reconciled whenever possible. Postmarketing exposure to patiromer was calculated by exposure in patient-years (PYs). Reporting rates of AEs were then calculated based on the total number of patients exposed in a specific postmarketing period and identified as the number of events per PY and multiplied by 100 to provide the percent events per $100 \mathrm{PYs}$. PYs are calculated as the number of patients exposed during the analysis period divided by 12 (based on the assumption that the length of patiromer treatment is 30 days). Reporting rate is the number of AEs per $100 \mathrm{PYs}$ and is calculated as total number of AEs divided by PYs $\times 100$. For the global pharmacovigilance database, reporting rates (\% of events per $100 \mathrm{PYs}$ ) are reported. For the clinical trial program $(N=666)[10$, $11,15,16]$, the frequencies ( $\%$ of patients with the event/all patients exposed) are reported. A common pharmacovigilance database limitation is that no accurate denominator is used because of the constant evolution of reports received daily. The denominator used here was determined by setting data cut-off dates, and no changes, additions, or updates to the data were made.

\section{Results}

Between January 2016 and September 2019, there were an estimated 45,000 PYs of exposure to patiromer. The total number of ICSRs in the global drug safety and pharmacovigilance database was 17,823 , of which 10,970 (62\%) were from solicited sources and 5966 (23\%) were medically confirmed (reported by a healthcare provider). The total number of AEs in the database was 38,109. Overall, 95\% of the patients with AEs were treated in the USA; 9981 (56\%) were male, 7664 (43\%) were female, and 178 (1\%) had unknown gender; 10,298 patients $(58 \%)$ were aged $>65$ years. The source of the AEs was primarily solicited (62\%), whereas $38 \%$ were unsolicited reports (spontaneous). Of the AE cases, $26 \%$ were serious AEs (45\% medically confirmed) and $74 \%$ were nonserious AEs (30\% medically confirmed). Overall, the vast majority of cases had limited information, such as the start and stop date of AEs and patiromer treatment, laboratory parameters, causality, etc., so a complete medical evaluation could not be performed. All parameters in an FDA MedWatch form are collected in the pharmacovigilance database. The reporters are only required to give four standard parameters, which include patient identification, drug name, the AE, and reporter identification. Stringent follow-up is performed to collect more details, but the success rate is typically low.

\subsection{Deaths}

During the postmarketing survey period, 1214 deaths (73\% medically confirmed) were reported, corresponding to a cumulative annualized mortality rate of 2.69 per 100 PYs. Of note, no cause was reported for $80 \%$ of the deaths. As, in most cases, no further information was obtainable, these deaths could not be medically evaluated by the sponsor. By comparison, in the clinical trial program, 18 deaths were recorded in patients receiving patiromer, resulting in an annualized mortality rate of 5.81 per $100 \mathrm{PYs}$.

Table 1 lists cases with a reported cause of death $\geq 0.5 \%$ in the global pharmacovigilance database. The majority were in the cardiac disorders class (myocardial infarction, cardiac arrest, cardiac failure, cardiac disorders, congestive cardiac failure). This is consistent with the clinical trial program, in which the adjudicated cause of death in 14 of 18 patients were in the cardiac disorders class (sudden cardiac death, $\mathrm{n}=10$; acute myocardial infarction, $n=4$ ). The second most common class of AEs leading to death were renal and urinary disorders (renal failure, end-stage renal disease), whereas no adjudicated death occurred in the clinical trial program in the renal or urinary disorders class.

Table 1 Most common ${ }^{a}$ reported causes of death in the global pharmacovigilance database

\begin{tabular}{ll}
\hline Events & $\begin{array}{l}\text { Pharmacovigi- } \\
\text { lance reporting } \\
\text { rate }^{\text {b }}\end{array}$ \\
\hline Renal failure & $34(2.80)$ \\
Myocardial infarction & $33(2.70)$ \\
Cardiac arrest & $26(2.14)$ \\
Cardiac failure $^{\mathrm{c}}$ & $15(1.23)$ \\
Cardiac disorders $_{\text {Congestive cardiac failure }}^{\mathrm{d}}$ & $10(0.82)$ \\
Cerebrovascular accident $^{\text {End-stage renal disease }}$ & $10(0.82)$ \\
\hline
\end{tabular}

Data are presented as $\mathrm{n}(\%)$

$P Y$ patient-years

${ }^{\text {a Reported in }} \geq 0.5 \%$ of 1214 deaths. Of the 1214 reported deaths in the patiromer global pharmacovigilance database, $80 \%$ had no reported cause

${ }^{\mathrm{b}}$ Reporting rate $=(\#$ events/\# PY $[45,000]) \times 100=\%$ of events per 100 PYs. Between January 2016 and September 2019, there were an estimated 45,000 PYs of exposure to patiromer in the global pharmacovigilance database

${ }^{\mathrm{c}}$ Includes verbatim terms of heart failure, cardiac failure, cardiac failure (not otherwise specified), cardiac function failure, and cardiac failure aggravated

${ }^{\mathrm{d}}$ Includes verbatim terms of congestive heart failure, congestive cardiac failure, cardiac failure congestive, and cardiac failure congestive aggravated 


\subsection{Most Common Serious Adverse Events (AEs)}

Overall, $6 \%$ of serious AEs in the global pharmacovigilance database met the criteria of hospitalization. Serious AEs with a reporting rate of $\geq 0.5 \%$ were dialysis $(0.69 \%$; $39.4 \%$ medically confirmed) and pneumonia ( $0.58 \%$; $40.6 \%$ medically confirmed). By comparison, there was no report of serious AEs of dialysis in the clinical trial program (patients on dialysis or with a potential need for dialysis were excluded from these clinical trials). Serious AEs of pneumonia were observed in $0.30 \%$ of patients in the clinical trial program. There is no mechanistic causality for these two events, as patiromer is not absorbed, but the treated patient population is predominantly renally impaired. Table 2 shows the most common serious AEs in the clinical trial program (with a reporting rate of $\geq 0.5 \%$ ) and corresponding rates in the global pharmacovigilance database.

Events of intestinal necrosis and other serious gastrointestinal AEs of special interest per the EMA had a reporting rate of $<0.05 \%$. These events are included in a biannual analysis for the potential risk of severe gastrointestinal disorders, including intestinal necrosis and perforation. The current analysis concluded that there is no evidence that patiromer caused severe gastrointestinal disorders, such as gastrointestinal ischemia, necrosis, or perforation.

Table 2 Serious adverse events reported in the patiromer clinical trial program with a frequency of $\geq 0.5 \%$ and corresponding reporting rates in the global pharmacovigilance database

\begin{tabular}{|c|c|c|}
\hline Serious AEs ${ }^{\mathrm{a}}$ & $\begin{array}{l}\text { Clinical trial } \\
\text { program }^{\mathrm{b}}\end{array}$ & $\begin{array}{l}\text { Pharmacovigi- } \\
\text { lance reporting } \\
\text { rate }^{\mathrm{c}}\end{array}$ \\
\hline Chronic renal failure & $7(1.1)$ & $0(0.0)$ \\
\hline Sudden death & $6(0.9)$ & $0(0.0)$ \\
\hline Sudden cardiac death & $5(0.8)$ & $2(0.0)$ \\
\hline Acute myocardial infarction & $4(0.6)$ & $3(0.0)$ \\
\hline Myocardial infarction & $3(0.5)$ & $120(0.2)$ \\
\hline Atrial fibrillation & $3(0.5)$ & $45(0.1)$ \\
\hline Cardiac failure & $3(0.5)$ & $45(0.1)$ \\
\hline
\end{tabular}

Data are presented as $n(\%)$

AEs adverse events, $P Y$ patient-years

${ }^{a}$ Overall, $6 \%$ of serious AEs met the criteria of hospitalization

${ }^{\mathrm{b}}$ Safety population from the patiromer clinical trial program $(N=$ 666)

${ }^{\mathrm{c}}$ Reporting rate $=(\#$ events/\# PY [45,000] $) \times 100=\%$ of events per 100 PYs. Between January 2016 and September 2019, there were an estimated 45,000 PYs of exposure to patiromer in the global pharmacovigilance database

\subsection{Most Common Nonserious AEs}

Consistent with the clinical trial program, the most commonly reported clinical nonserious AEs in the global pharmacovigilance data were gastrointestinal (Table 3). For the two most common nonserious AEs, constipation and diarrhea, the reporting rates were 6.90 and $3.48 \%$ in the global pharmacovigilance database and 7.2 and $4.8 \%$ of patients in the clinical trial program.

In addition to lowering serum potassium, patiromer use has been associated with decreases in serum magnesium. Because patiromer exchanges calcium for potassium, increases in serum calcium are of interest. Serum potassium values $<3.5 \mathrm{mmol} / \mathrm{L}$ were reported in $4.7 \%$ of patients in the clinical trial program. "Blood potassium decreased" (the term captured in the global pharmacovigilance database) had a postmarketing reporting rate of $0.45 \%$. AEs of hypomagnesemia and "blood magnesium decreased" were observed in 5.3 and $0.8 \%$ of patients in the clinical trial program and $0.02 \%$ and $0.16 \%$, respectively, in the global pharmacovigilance database. Hypercalcemia was observed in a single patient $(0.2 \%)$ in the clinical trial program, and there was no AE of "blood calcium increased." AEs of hypercalcemia and "blood calcium increased" had a reporting rate of 0.06 and $0.09 \%$, respectively, in the global pharmacovigilance database.

Hypersensitivity reactions (all mild to moderate) were reported in $0.3 \%$ of patients receiving patiromer in the clinical trial program; reactions included edema of the lips. Hypersensitivity reactions had a reporting rate of $0.29 \%$ (131 events; 44 [33.5\%] medically confirmed); of these, 24

Table 3 Most common ${ }^{\mathrm{a}}$ gastrointestinal adverse events in the patiromer clinical trial program and corresponding rates in the global pharmacovigilance database

\begin{tabular}{lll}
\hline Nonserious AEs & $\begin{array}{l}\text { Clinical trial } \\
\text { program }^{\mathrm{b}}\end{array}$ & $\begin{array}{l}\text { Pharmacovigi- } \\
\text { lance reporting } \\
\text { rate }^{\mathrm{c}}\end{array}$ \\
\hline Constipation & $48(7.2)$ & $3105(6.90)$ \\
Diarrhea & $32(4.8)$ & $1566(3.48)$ \\
Nausea & $15(2.3)$ & $755(1.67)$ \\
Abdominal discomfort & $13(2.0)$ & $647(1.43)$ \\
Flatulence & $13(2.0)$ & $568(1.26)$
\end{tabular}

Data are presented as $n(\%)$

$A E$ adverse events, $P Y$ patient-years

${ }^{a}$ Occurring in $\geq 2 \%$ of patients in the safety population of the patiromer clinical trial program

${ }^{\mathrm{b}} N=666$

${ }^{\mathrm{c}}$ Reporting rate $=(\#$ events/\# PY $[45,000]) \times 100=\%$ of events per 100 PYs. Between January 2016 and September 2019, there were an estimated 45,000 PYs of exposure to patiromer in the global pharmacovigilance database 
were categorized as serious (five [20.8\%] medically confirmed) in the global pharmacovigilance database.

Finally, drug-drug interactions (DDIs) were closely monitored because of the recommended 3-h separation from administration of other oral medications. Most of the reported DDI events occurred during the first 2 years after US marketing approval, and the majority involved consumer inquiries about whether a possible DDI existed. A search of the global pharmacovigilance database was performed for DDIs, which includes reported events of drug interaction, inhibitory drug interaction, and potentiating drug interaction. DDI events had a reporting rate of $0.1 \%$ (48 events, three serious, and 12 medically confirmed).

\section{Discussion}

This manuscript presents the first global postmarketing safety survey of the sodium-free nonabsorbed potassium binder patiromer (Veltassa ${ }^{\circledR}$ ) since its approval for the treatment of hyperkalemia in October 2015 in the USA [1] and in May 2017 in the EU [2], using the global pharmacovigilance database of a patiromer manufacturer. In the current analysis, with data collected from the beginning of 2016 through September 2019, there were approximately 45,000 PYs of exposure (largely US patients) to this novel potassium binder. The overall mortality rate in this "real-life" nonselected population (2.69 per $100 \mathrm{PYs}$ ) was lower than that observed in the clinical trial program of patiromer (5.81 per 100 PYs). In the global pharmacovigilance database, $67 \%$ of patients were aged $>65$ years. In the clinical trials, $59.8 \%$ of patients were aged $\geq 65$ years and $93 \%$ of patients had CKD. The demography of patients in the pharmacovigilance database is consistent with the epidemiology of hyperkalemia, in that older patients are affected more frequently. In addition, older patients are more likely to have hypertension, diabetes, and $\mathrm{HF}$ requiring RAASi therapy. It seems prudent that all patients with CKD—and most crucially older patientsparticularly those on RAASi medications, have their serum potassium levels monitored regularly.

Overall, $80 \%$ of deaths in the global pharmacovigilance database had no reported cause and were obtained mostly from solicited sources, which typically are collected on a monthly or more frequent basis. A medical evaluation by the sponsor concerning causality is not possible when very minimal information is provided. We can ascertain, in some cases-when medical history includes multiple comorbidities or concomitant medications (signifying an underlying condition such as diabetes, CKD, hypertension, cardiac disorders, hyperlipidemia, etc.) - that the cause of death could be confounded by multiple risk factors, similar to what was seen in the clinical studies.
A biannual analysis is performed for all cases with an outcome of death and is submitted to the health authorities in the form of an aggregate safety report. The conclusion of all analyses to date is that the data showed no new safety concerns and that the benefit-risk profile of patiromer remained positive and unchanged, with no objection from health authorities.

The total number of AEs reported with patiromer was similar between the solicited and unsolicited reports. Consistent with the clinical trial program results, the most frequent AEs were gastrointestinal and reported as nonserious in most instances. Of importance, in the clinical trial program, constipation generally resolved during the course of treatment; however, this information is not reported consistently in the postmarketing setting.

Events of intestinal necrosis and other serious gastrointestinal AEs of special interest per the EMA had a reporting rate of $<0.05 \%$. A biannual analysis is performed for all cases for the potential risk of severe gastrointestinal disorders, including intestinal necrosis and perforation, because of reports of such events for SPS and CPS, and submitted to the health authorities in the form of an aggregate safety report. The current analysis concluded that there was no evidence that patiromer caused severe gastrointestinal disorders, such as gastrointestinal ischemia, necrosis, or perforation. The analysis supported that often multiple confounding factors may be considered as alternative etiologies. Overall, there were no new gastrointestinal safety concerns, and the benefit-risk profile of patiromer remains positive and unchanged, with no objection from health authorities.

The frequency of the event "blood potassium decreased" in the postmarketing setting was low (0.45\% of the AEs) and typically was not serious. This low rate is reassuring since a U-shape relationship was consistently observed between serum potassium and death across cohorts (gathered from general populations and patients with hypertension, CKD, or acute or chronic HF [23]), with both hypokalemia and hyperkalemia being associated with worse outcomes [3, 24]. An AE of specific interest with patiromer because of its mechanism of action is hypomagnesemia. During treatment with patiromer, both potassium and magnesium should be monitored, and supplementation with magnesium should be considered for patients who develop low serum magnesium levels with patiromer [1]. The frequency of reported hypomagnesemia or "blood magnesium decreased" was low in the postmarketing setting.

Calcium rather than sodium was chosen as the counterexchange ion in patiromer primarily because high sodium intake augments cardiorenal risk, either by leading to hypertension or by reducing the efficacy of RAASi [25]. In addition, treatment guidelines restrict sodium intake in patients with hypertension, CKD, and HF; the American Heart Association [26] currently recommends a dietary 
sodium intake of $<1.5 \mathrm{~g}$ per day for patients with these disorders, who are most at risk for hyperkalemia. An 8.4 $\mathrm{g}$ dose of patiromer contains $1.6 \mathrm{~g}$ of calcium. Studies in healthy volunteers [27] and patients with CKD [28] suggested that patiromer was associated with a modest increase in 24-h urine calcium. Overall, no changes in mean serum calcium were seen, but patients exhibited small but significant decreases in intact parathyroid hormone (iPTH), along with reduced serum 1,25-dihydroxyvitamin D [28]. As a marker of calcium load, the decrease in iPTH suggests that some of the calcium released by patiromer is absorbed. It also appears that some of the calcium released by patiromer in the gastrointestinal tract binds to phosphate, leading to the formation of insoluble salts that are excreted in the stool. In a subgroup of patients with concomitant hyperphosphatemia, serum and urine phosphate levels decreased with patiromer treatment [28].

The potential occurrence of hypercalcemia was thoroughly assessed in the clinical trial program and was observed in a single patient. In the postmarketing setting, hypercalcemia or "blood calcium increased" had low reporting rates of 0.06 and $0.09 \%$ per 100 PYs. Hypercalcemia is not uncommon in patients with CKD [29], for instance, when supplemented with vitamin D for secondary hyperparathyroidism.

\subsection{Limitations}

Several limitations with the global pharmacovigilance database are inherent to postmarketing data, such as limited information on demographics, medical history, posology, laboratory results, start date of drug and event, and health professional assessment of causal relationship. We also acknowledge the potential for under-reporting of AEs in the global pharmacovigilance database, based on the assumptions made in calculating the PYs of exposure. However, the majority of cases (62\%) were obtained from solicited programs (which, in the case of the patient-support program, contacted prescribed patients monthly or more often depending on the prescription). Any AE, regardless of causal relationship, that was mentioned during this communication was captured in the pharmacovigilance database. Therefore, the true picture of a product's safety may only emerge over the years that make up a product's lifetime in the marketplace [30].

\section{Conclusions}

The results of this large postmarketing survey using all reported AEs confirm that this novel potassium binder has a safety profile similar to that observed in clinical trials. Patiromer's efficacy in controlling hyperkalemia was previously demonstrated [10] across the development program. New patiromer trials, currently ongoing (DIAMOND) [31] and newly initiated (PLATINUM) [32], will provide additional safety information on the use of patiromer to enable the use of RAASi in the guideline-directed management of HF with reduced ejection fraction and as adjunctive therapy for management of severe hyperkalemia in the emergency room, respectively.

Acknowledgments Editorial support services were provided by Impact Communication Partners, Inc., and funded by Vifor Pharma, Inc.

\section{Declarations}

Funding The analysis reported in this manuscript were funded by Vifor Pharma, Inc.

Conflicts of interest Patrick Rossignol has consulted for Bayer, G3P, Idorsia, and KBP; has received honoraria from Ablative Solutions, AstraZeneca, Bayer, Boehringer Ingelheim, Corvidia, CVRx, Fresenius, Grunenthal, Novartis, Novo Nordisk, Vifor Pharma, Inc., Sanofi, Sequana Medical, Servier, Stealth Peptides, and Vifor Fresenius Medical Care Renal Pharma; and is a cofounder of CardioRenal. Lea David, Christine Chan, and Ansgar Conrad are employed by and own stock in Vifor Pharma, Inc. Matthew R. Weir has consulted for and received honoraria for scientific advisory boards from Vifor Pharma, Inc., Vifor Pharma Management Ltd., AstraZeneca, Bayer, Boehringer Ingelheim, Janssen, MSD, and Novo Nordisk.

Availability of data and material The datasets generated and analyzed for this study are from the global pharmacovigilance database of Vifor Pharma, Inc. and are not publicly available. Patiromer postmarketing reports are submitted to the FDA and can be obtained via the FDA Adverse Event Reporting System (FAERS), a public database that contains information on AEs and medication error reports submitted to the FDA.

Ethics approval All studies included in this analysis were conducted in accordance with the ethical principles that comply with the Declaration of Helsinki and are consistent with good clinical practices and applicable regulatory requirements.

Consent Not applicable.

Author contributions All authors contributed to the conception and design of the analysis as well as the interpretation of data and critical review of this manuscript. All authors approved the manuscript for submission to the journal. The authors assume responsibility and accountability for data integrity and accuracy.

Open Access This article is licensed under a Creative Commons Attribution-NonCommercial 4.0 International License, which permits any non-commercial use, sharing, adaptation, distribution and reproduction in any medium or format, as long as you give appropriate credit to the original author(s) and the source, provide a link to the Creative Commons licence, and indicate if changes were made. The images or other third party material in this article are included in the article's Creative Commons licence, unless indicated otherwise in a credit line to the material. If material is not included in the article's Creative Commons licence and your intended use is not permitted by statutory regulation or exceeds the permitted use, you will need to obtain permission 
directly from the copyright holder. To view a copy of this licence, visit http://creativecommons.org/licenses/by-nc/4.0/.

\section{References}

1. Veltassa ${ }^{\circledR}$ (patiromer) for oral suspension [package insert]. Redwood City, CA: Relypsa, Inc. 2018. https://www.veltassa.com/pi. pdf.

2. European Medicines Agency. Veltassa (patiromer). https://www. ema.europa.eu/en/medicines/human/EPAR/veltassa. Updated 19 June 2019. Accessed 1 July 2020.

3. Rossignol P. A new area for the management of hyperkalaemia with potassium binders: clinical use in nephrology. Eur Heart J Suppl. 2019;21(Suppl A):A48-54. https://doi.org/10. 1093/eurheartj/suy032 (Erratum in: JAMA Intern Med. 2020;180(4):618).

4. Noel JA, Bota SE, Petrcich W, Garg AX, Carrero JJ, Harel Z, et al. Risk of hospitalization for serious adverse gastrointestinal events associated with sodium polystyrene sulfonate use in patients of advanced age. JAMA Intern Med. 2019;179(8):1025-33. https:// doi.org/10.1001/jamainternmed.2019.0631.

5. Laureati P, Xu Y, Trevisan M, Schalin L, Mariani I, Bellocco $\mathrm{R}$, et al. Initiation of sodium polystyrene sulphonate and the risk of gastrointestinal adverse events in advanced chronic kidney disease: a nationwide study. Nephrol Dial Transplant. 2020;35(9):1518-26. https://doi.org/10.1093/ndt/gfz150.

6. LOKELMA $^{\circledR}$ (sodium zirconium cyclosilicate) for oral suspension [package insert]. Wilmington, DE: AstraZeneca Pharmaceuticals LP. 2020. https://www.azpicentral.com/lokelma/lokelma.pdf\# page $=1$. Accessed 1 July 2020 .

7. Whelton PK, Carey RM, Aronow WS, Casey DE Jr, Collins KJ, Himmelfarb CD, et al. ACC/AHA/AAPA/ABC/ACPM/AGS/ APhA/ASH/ASPC/NMA/PCNA guideline for the prevention, detection, evaluation, and management of high blood pressure in adults: executive summary: a report of the American College of Cardiology/American Heart Association Task Force on Clinical Practice Guidelines. Hypertension. 2018;71:1269-324. https://doi. org/10.1161/HYP.0000000000000066.

8. American Diabetes Association. 10. Cardiovascular disease and risk management: standards of medical care in diabetes-2020. Diabetes Care. 2020;43(Suppl 1):S111-34. https://doi.org/10. 2337/dc20-S010.

9. Disease K. Improving global outcomes (KDIGO) Blood Pressure Work Group-KDIGO Clinical Practice Guideline for the Management of Blood Pressure in Chronic Kidney Disease. Kidney Int Suppl. 2012;2(5):337-414.

10. Weir MR, Bakris GL, Bushinsky DA, Mayo MR, Garza D, Stasiv Y, et al. Patiromer in patients with kidney disease and hyperkalemia receiving RAAS inhibitors. N Engl J Med. 2015;372:211-21. https://doi.org/10.1056/NEJMoa1410853.

11. Pitt B, Anker SD, Bushinsky DA, Kitzman DW, Zannad F, Huang IZ. Evaluation of the efficacy and safety of RLY5016, a polymeric potassium binder, in a double-blind, placebo-controlled study in patients with chronic heart failure (the PEARL-HF) trial. Eur Heart J. 2011;32:820-8. https://doi.org/10.1093/eurheartj/ehq502.

12. Agarwal R, Rossignol P, Romero A, Garza D, Mayo MR, Warren $\mathrm{S}$, et al. Patiromer versus placebo to enable spironolactone use in patients with resistant hypertension and chronic kidney disease (AMBER): a phase 2, randomised, double-blind, placebocontrolled trial. Lancet. 2019;394(10208):1540-50. https://doi. org/10.1016/S0140-6736(19)32135-X.

13. Seferovic PM, Ponikowski P, Anker SD, Bauersachs J, Chioncel $\mathrm{O}$, Cleland JGF, et al. Clinical practice update on heart failure
2019: pharmacotherapy, procedures, devices and patient management-an expert consensus meeting report of the Heart Failure Association of the European Society of Cardiology. Eur J Heart Fail. 2019;21(10):1169-86. https://doi.org/10.1002/ejhf.1531.

14. Disease K. Improving global outcomes (KDIGO) Diabetes Work Group-KDIGO 2020 Clinical Practice Guideline for Diabetes Management in Chronic Kidney Disease. Kidney Int. 2020;98(4S):S1-115. https://doi.org/10.1016/j.kint.2020.06.019.

15. Bakris GL, Pitt B, Weir MR, Freeman MW, Mayo MR, Garza D, et al. Effect of patiromer on serum potassium level in patients with hyperkalemia and diabetic kidney disease: the AMETHYST-DN randomized clinical trial. JAMA. 2015;314:151-61. https://doi. org/10.1001/jama.2015.7446.

16. Pitt B, Bushinsky DA, Kitzman DW, Ruschitzka F, Metra M, Filippatos G, et al. Evaluation of an individualized dose titration regimen of patiromer to prevent hyperkalaemia in patients with heart failure and chronic kidney disease. ESC Heart Fail. 2018;5(3):257-66. https://doi.org/10.1002/ehf2.12265.

17. Kovesdy CP, Gosmanova EO, Woods SD, Fogli JJ, Rowan CG, Hansen JL, et al. Real-world management of hyperkalemia with patiromer among United States Veterans. Postgrad Med. 2020;132(2):176-83. https://doi.org/10.1080/00325481.2019. 1706920.

18. Desai NR, Rowan CG, Alvarez PJ, Fogli J, Toto RD. Hyperkalemia treatment modalities: A descriptive observational study focused on medication and healthcare resource utilization. PLoS ONE. 2020;15(1):e0226844. https://doi.org/10.1371/journal.pone. 0226844.

19. European Medicines Agency (EMA). European Medicines Agency (2017b) Guideline on Good Pharmacovigilance Practices (GVP): Annex I —definitions (Rev 4). https://www.ema.europa. eu/en/documents/scientific-guideline/guideline-good-pharmacovi gilance-practices-annex-i-definitions-rev-4_en.pdf. Updated 9 October 2017. Accessed 1 July 2020.

20. US Office of the Federal Register and the Government Publishing Office. Part 314 - applications for FDA approval to market a new drug. In: Electronic code of federal regulations. 2020. https:// www.ecfr.gov/cgi-bin/text-idx?SID=3080e1d2e8c89a91ec01 $\mathrm{d} 1 \mathrm{c} 42 \mathrm{a} 5 \mathrm{ddde} 7 \& \mathrm{mc}=$ true $\&$ node $=\mathrm{se} 21.5 .314 \_180 \& \mathrm{rgn}=\operatorname{div} 8$. Updated 30 June 2020. Accessed 1 July 2020.

21. Uppsala Monitoring Centre (UMC). Glossary of pharmacovigilance terms. In: Global Pharmacovigilance. UMC. 2020. https:// www.who-umc.org/global-pharmacovigilance/publications/gloss ary/. Accessed 1 July 2020.

22. European Medicine Agency (EMA), Inspections, Human Medicines Pharmacovigilance and Committees Division. Important medical event terms list (MedDRA version 22.1). 16 September 2019. https://www.alims.gov.rs/ciril/files/2019/06/092_13_ 01316_2019_5_001_1_xlsx.pdf. Accessed 1 July 2020.

23. Rossignol P, Duarte K, Girerd N, Karoui M, McMurray JJV, Swedberg K, et al. Cardiovascular risk associated with serum potassium in the context of mineralocorticoid receptor antagonist use in patients with heart failure and left ventricular dysfunction. Eur J Heart Fail. 2020;22(8):1402-11. https://doi.org/10.1002/ ejhf.1724.

24. Kovesdy CP, Matsushita K, Sang Y, Brunskill NJ, Carrero JJ, Chodick G, et al. Serum potassium and adverse outcomes across the range of kidney function: a CKD Prognosis Consortium metaanalysis. Eur Heart J. 2018;39(17):1535-42. https://doi.org/10. 1093/eurheartj/ehy100.

25. Clegg DJ, Cody M, Palmer BF. Challenges in treating cardiovascular disease: restricting sodium and managing hyperkalemia. Mayo Clin Proc. 2017;92(8):1248-60. https://doi.org/10.1016/j. mayocp.2017.04.006.

26. Arnett DK, Blumenthal RS, Albert MA, Buroker AB, Goldberger ZD, Hahn EJ, et al. 2019 ACC/AHA guideline on the primary 
prevention of cardiovascular disease: executive summary: a report of the American College of Cardiology/American Heart Association Task Force on clinical practice guidelines. J Am Coll Cardiol. 2019;74:1376-414. https://doi.org/10.1016/j.jacc.2019.03.009.

27. Bushinsky DA, Spiegel DM, Gross C, Benton WW, Fogli J, Hill Gallant KM, et al. Effect of patiromer on urinary ion excretion in healthy adults. Clin J Am Soc Nephrol. 2016;11(10):1769-76. https://doi.org/10.2215/CJN.01170216.

28. Bushinsky DA, Spiegel DM, Yuan J, Warren S, Fogli J, Pergola PE. Effects of the potassium-binding polymer patiromer on markers of mineral metabolism. Clin J Am Soc Nephrol. 2019;14(1):103-10. https://doi.org/10.2215/CJN.04500418.

29. National Kidney Foundation (NKF) KDOQI Guidelines. Guideline 6: serum calcium and calcium-phosphorus product. In: KDOQI Clinical Practice Guidelines for Bone Metabolism and
Disease in Chronic Kidney Disease. http://kidneyfoundation. cachefly.net/professionals/KDOQI/guidelines_bone/guide6.htm. Accessed 1 July 2020.

30. U.S. Food \& Drug Administration (FDA). Step 5: FDA Post-Market Drug Safety Monitoring. In: The drug development process. https://www.fda.gov/patients/drug-development-process/step-5fda-post-market-drug-safety-monitoring. Updated 4 January 2018. Accessed 1 July 2020.

31. Patiromer for the Management of Hyperkalemia in Subjects Receiving RAASi Medications for the Treatment of Heart Failure (DIAMOND). Updated 20 May 2020. Accessed 1 July 2020.

32. Patiromer Utility as an Adjunct Treatment in Patients Needing Urgent Hyperkalemia Management (PLATINUM). Updated 24 June 2020. Accessed 1 July 2020. 